\title{
The Influence of Sad Mood on Cognition
}

\author{
Lara G. Chepenik \\ Yale University
}

\author{
Lauren A. Cornew \\ University of California, San Diego
}

\author{
Martha J. Farah \\ University of Pennsylvania
}

\begin{abstract}
Neuroimaging has identified an overlapping network of brain regions whose activity is modulated by mood and cognition. Studies of depressed individuals have shown changes in perception, attention, memory, and executive functions. This suggests that mood has a pervasive effect on cognition. Direct evidence of the effect of sad mood on cognition is surprisingly limited, however. Published studies have generally addressed a single cognitive ability per study because the fleeting nature of laboratory-induced mood precludes extended testing, and robust findings are limited to mood effects on memory for emotional stimuli. In this study, sad mood was induced and prolonged, enabling the effects of mood to be assessed for an array of abilities, including those that share neural substrates with sad mood and those affected by depression. Sad mood affected memory for emotional words and facial emotion recognition, but not the other processes measured, with a significant nonuniformity of effect over tasks. These results are consistent with circumscribed effects of sad mood on certain emotion-related cognitive processes, but not on cognition more generally.
\end{abstract}

Keywords: mood, cognition, mood induction, memory

How does mood affect cognition? Intuitively, it seems likely that mood influences what we think about and how efficiently our thought processes proceed. Consistent with this intuition, the functional neuroanatomy of mood and that of cognition encompass overlapping networks of cortical and subcortical brain regions (Cabeza \& Nyberg, 2000; Phan, Wager, Taylor, \& Liberzon, 2002). Also consistent is the finding that clinical depression may be accompanied by a number of cognitive abnormalities, including biases in the content of perception, attention and memory toward negative affective content, and impairments in the speed and accuracy of emotionally neutral cognitive processes, particularly executive functions.

Three types of evidence suggest that the substrates of cognition are shared with, or open to extensive influence by, the substrates of mood. The first comes from functional neuroimaging. The other two come from behavioral studies of cognition. These include studies of the effects of depression on cognition, already mentioned, and of the effects of laboratory-induced moods on the cognitive processes of normal healthy participants.

Lara G. Chepenik, Mood Disorders Research Program, Department of Psychiatry, Yale University; Lauren A. Cornew, Department of Psychology, University of California, San Diego; Martha J. Farah, Center for Cognitive Neuroscience, Department of Psychology, University of Pennsylvania.

The research reported here was supported by National Institutes of Health Grants R21-DA01586, R01-HD043078, and R01-DA18913.

Correspondence concerning this article should be addressed to Lara G. Chepenik, Department of Psychiatry, Yale University, 300 George Street, Suite 901, New Haven CT 06511. E-mail: lara.chepenik@ yale.edu
Functional neuroimaging studies have indicated that the anatomical basis of sad mood and cognitive processing have considerable overlap. Sad mood, in both healthy participants and those with clinical depression, has been shown to influence the activity of a common set of prefrontal and limbic brain regions (Davidson, Pizzagalli, Nitschke, \& Putnam, 2002; Mayberg et al., 1999). Many of these are also implicated in cognitive functions. The overlap between the brain areas modulated by sad mood and cognition is consistent with a shared, interactive neural architecture for cognition and mood.

For example, activity in the prefrontal cortex is altered both in normal sad mood and in depression (for reviews, see Davidson et al., 2002; Mayberg et al., 1999; Murphy, Nimmo-Smith, \& Lawrence, 2003; Phan et al., 2002; M. L. Phillips, Drevets, Rauch, \& Lane, 2003a, 2003b). This brain region is also associated with the set of higher order cognitive abilities referred to as executive functions. More specifically, dorsolateral prefrontal cortex, which is reliability activated by working memory tasks (Braver et al., 1997; Casey et al., 1998; Cohen et al., 1997; Smith \& Jonides, 1997), has been found to be hypoactive in depression (for review, see Drevets, 1999). Studies of induced sad mood in healthy volunteers have also found abnormally low activation in this area (Aalto et al., 2002; Gemar, Kapur, Segal, Brown, \& Houle, 1996; Liotti et al., 2000; Mayberg et al., 1999). Medial regions of the prefrontal cortex, including the anterior cingulate cortex, which play a role in cognitive control and inhibitory processing (Bush, Luu, \& Posner, 2000; Kiehl, Liddle, \& Hopfinger, 2000; Liddle, Kiehl, \& Smith, 2001; Peterson et al., 1999), also show abnormal levels of activation in both depression (Bench et al., 1992; Drevets et al., 1992; Kennedy et al., 2001) and normal sadness (for reviews, see Phan et al., 2002). A recent meta-analysis by Steele and Lawrie (Steele \& Lawrie, 2004) mapped the activation maxima 
from published studies of cognitive tasks and emotion induction tasks, all in healthy participants, onto a common brain space to assess the degree of segregation and overlap. They found that the "center of gravity" of the emotional maxima was located more inferiorly than that of the cognitive maxima, but that the overlap between the two distributions was extensive, encompassing far more territory than either type of activation alone.

Another important nexus of cognition and mood in the brain is the amygdala, which plays a role in memory, perception, and mood. Functional neuroimaging studies of normal healthy participants have shown that circuits within the amygdala mediate both the encoding of emotionally valenced material into memory (see Phelps \& LeDoux, 2005, for review) and the perception of facial emotion (see Haxby, Hoffman, \& Gobbini, 2002, for a review). Functional neuroimaging studies have also shown that the amygdala is hyperactive in depression (for review, see Drevets, 2003); this has also been shown in many studies of normal sad mood (Aalto et al., 2002; Eugene et al., 2003; Lane, Reiman, Ahern, Schwartz, \& Davidson, 1997; Levesque et al., 2003).

On the basis of the distributed and overlapping substrates of sad mood and cognition, one would expect sad mood to have extensive effects on cognitive processing. At first glance, behavioral studies of sad mood and cognition support this prediction. A substantial literature exists on mood effects on memory in depressed and normal participants. In general, depressed patients are more likely to recall negatively valenced words, story facts, and autobiographical memories in laboratory contexts compared with healthy control participants (see Williams, Watts, Macleod, \& Mathews, 1997, for a review). A similar pattern of bias toward negative memory has been reported in normal participants after laboratory sadness induction (Bower \& Forgas, 2001).

Executive functions, including working memory and cognitive control, are frequently (although not invariably) found to be impaired in depressed patients (for reviews, see Austin et al., 1999; Marvel \& Paradiso, 2004; Rogers et al., 2004). These demonstrations have included tasks that stress working memory, the capacity to hold a stimulus representation in mind over a brief interval of time and avoid interference from subsequent stimuli. The Digit Span subtest of the Wechsler Adult Intelligence Scale (WAIS; Wechsler, 1997) IQ test is a well-known working memory task in which participants must retain a sequence of auditorily presented digits long enough to repeat them back in either the original or a reversed order and on which depressed patients perform below controls (Moritz et al., 2002). The 2-back task was developed as a test of working memory in functional neuroimaging studies and requires the participant to continually encode new items into working memory, comparing each new item with the one presented two items earlier. Depression also lowers performance on this measure of working memory (Harvey et al., 2004). Cognitive control, the process by which the needed amount of attention is allocated to avoid errors, is also impaired in depression, as measured by tasks such as the Stroop Color and Word Test (Rogers et al., 2004) and Go/No-Go Association Task (Langenecker et al., 2005).

Facial emotion perception is altered in depression. Depressed patients are slower and less accurate in classifying facial emotions (Bouhuys, Geerts, \& Gordijn, 1999; Cooley \& Nowicki, 1989; Feinberg, Rifkin, Schaffer, \& Walker, 1986; Gur et al., 1992; Langenecker et al., 2005; Leppanen, Milders, Bell, Terriere, \&
Hietanen, 2004; Mikhailova, Vladimirova, Iznak, Tsusulkovskaya, \& Sushko, 1996). Most studies have not found bias toward the perception of negative emotions in addition to general impairment, and when found, bias appears to be correlated with patients' comorbid anxiety rather than with sadness (Bouhuys, Geerts, \& Mersch, 1997). There is little corresponding literature on facial emotion perception and sad mood in normal participants. Bouhuys, Bloem, and Groothuis (1995) reported a study in which facial emotion recognition was compared in normal healthy adults after sad and happy mood inductions. Although no general effect of sad mood was observed, when the data from the half of the participants who responded most strongly to the induction were analyzed separately, a bias toward sadness and rejection was noted for the subset of emotionally ambiguous faces.

Depression is also accompanied by attentional biases in favor of negatively valenced emotional material. This has been demonstrated using the "emotional Stroop" task (Williams, Mathews, \& MacLeod, 1996) and attention probe paradigms (MacLeod, Mathews, \& Tata, 1986). In the emotional Stroop task, slowing of color naming for emotionally valenced words is the measure of attentional bias. Depressed individuals display interference in the emotional Stroop (Lim \& Kim, 2005). Less work has investigated emotional Stroop performance as a function of mood in nondepressed participants, and results have been mixed. Stroop interference has been observed for sad words after sad mood induction in one study (Gilboa-Schechtman, Revelle, \& Gotlib, 2000), but not in another (Perez, Rivera, Fuster, \& Rodriguez, 1999).

Even when the emotional Stroop task yields positive results, their interpretation may be ambiguous (Algom, Chajut, \& Lev, 2004; McKenna \& Sharma, 2004), leading some researchers to seek a different measure of attentional bias for emotionally valenced stimuli. In the attention probe task, pairs of words are briefly presented on a screen followed by a simple visual task such as speeded dot detection. Task performance is facilitated at the location previously occupied by the word to which the participant attended. Depressed participants show relatively more attention to locations occupied by negatively valenced words than do nondepressed participants (B. P. Bradley, Mogg, \& Lee, 1997; Mathews, Ridgeway, \& Williamson, 1996; Mogg, Mathews, Eysenck, \& May, 1991). Only two studies have examined the effects of normal sad mood on attentional allocation in this type of task, and they found inconsistent results. The first found the predicted negative bias in just one of the three word-exposure durations tested (B. P. Bradley et al., 1997), and the second found a trend among participants without a history of depression toward paradoxically less negative bias in the negative mood condition relative to the neutral condition (McCabe, Gotlib, \& Martin, 2000).

This brief review shows that the literatures on the effects of normal and depressed sad mood on cognition diverge in the following respect: Whereas depression has been found in numerous studies to exert a fairly consistent influence on perception, attention, memory, and executive function, published reports of the effects of experimentally induced sad moods in healthy participants are much fewer in number, include more null results, and on balance consist mainly of biases in memory. This raises the possibility that sad mood per se does not exert an effect on cognition in general and that factors other than mood may be responsible for the cognitive changes seen in depression. According to this hypothesis, the absence of published research on the effects of 
normal mood on cognitive functions other than memory is due either to lack of study or to the file-cabinet effect, the tendency of null results to go unreported.

One reason that few studies of sad mood effects have been carried out across a broad range of normal cognition processes is a practical problem with laboratory mood induction studies. In contrast to depressed participants, whose sad mood is continual, normal participants' sad moods are fleeting, and this imposes a serious limitation on research. Mood induction procedures typically involve watching a sad film clip or recalling a sad life event, often while listening to sad music, and the mood they produce is typically experienced for less than $10 \mathrm{~min}$ (Fiedler, Nickel, Muehlfriedel, \& Unkelbach, 2001; Gilboa, Roberts, \& Gotlib, 1997; Seibert \& Ellis, 1991; Varner \& Ellis, 1998). The short-lived nature of experimentally induced sad mood in normal participants makes it difficult to assess the effects of sadness in more than one task at a time.

A limitation on the number of cognitive processes that can be studied in a single experiment would reduce the number of different cognitive processes investigated overall. In addition, a null result with a single task is difficult to interpret and is more likely to end up in the file cabinet, unreported, because in such cases one cannot know whether the mood induction was simply ineffective. In contrast, a null result in one task coupled with a positive result in another task suggests that the mood induction was effective in changing mood.

The experiments described here were undertaken to discriminate between the alternative relations between cognition and mood. They were designed to answer the question Does sad mood in normal healthy people affect the full range of cognitive abilities suggested by both the depression literature and the overlap between mood-related and cognition-related activation in the functional neuroimaging literature? Or, alternatively, does sad mood merely bias memory, without the more extensive effects on cognition seen in depressed patients?

The tasks used in the present study were selected to assess the range of neurocognitive abilities altered or impaired by depression, namely working memory, cognitive control, and recognition of emotional facial expressions, as well as a tendency to attend to and remember negatively valenced emotional material. We selected seven tasks to assess these abilities.

Perception, attention, and memory were assessed with the following: a facial expression perception task with multiple-choice oral-verbal response; an attention probe task in which attention to words of varying emotional valence is probed by means of a simple visual pattern discrimination task with button-press response; and a memory task in which words of varying emotional valence were presented visually for study and later tested by free recall and an "old-new" recognition test. The executive functions of working memory and cognitive control were assessed with two tasks each, to maximize the likelihood of finding evidence of mood effects. These tasks were the Object 2-Back task, a working memory task with nonverbalizable visual stimuli and button-press responses; the Digit Span task, a working memory task with verbal stimuli and oral-verbal responses; the Go/No-Go task, a cognitive control task with single-digit stimuli and button-press responses; and the Stroop Color-Word Interference task, a cognitive control task with verbal stimuli and oral-verbal responses.
To maximize the interpretability of any null results we might find with some of these abilities, we assessed them together with the same participants in the same testing sessions. Provided we were able to replicate the previously observed memory bias for sad material, we could conclude that our mood manipulation was effective. We prolonged the induced mood with follow-up mood inductions ("mood boosters") interposed between tasks and counterbalanced the order of tasks relative to mood inductions. We also maximized the sensitivity of the experiment by using a withinparticipant experimental design in which the same participants underwent a sad mood induction on one day and a neutral mood induction on a different day.

\section{Method}

\section{Participants}

Thirty-three healthy volunteers were recruited for this study. The group consisted of 14 men and 19 women, with a mean age of 25.28 years $(S D=11.14$, range $=18-56)$. Participants responded to flyers and e-mail advertisements posted on the University of Pennsylvania campus and e-mail newsgroups. Exclusion criteria included past or present history of psychiatric illness, substance abuse, head injury, or use of medications that might affect cognitive function. Inclusion criteria consisted of at least a partial college education (mean years of education $=15.30, S D=1.94$, range $=12-20$ ) and English as a first language, to narrow the variability of performance across participants and to ensure familiarity with the vocabulary used in the memory test. All participants were tested under both a neutral and a sad mood induction (16 received the neutral induction first, 17 received the sad induction first). Mean incoming Beck Depression Inventory scores were 2.6 $(S D=2.3$, range $=0-7)$ on the neutral mood induction day and $3.9(S D=3.4$, range $=0-7)$ on the sad mood induction day (see Figure 1). Both of a participant's testing sessions were scheduled at the same time of day to control for diurnal changes in cognition.

\section{Procedure}

Mood induction. Mood-congruent music was played while a mood induction procedure script was read to the participant (1-2 $\mathrm{min})$. The investigator then stepped out of the room for $5 \mathrm{~min}$, giving the participants instructions to focus on the imagery suggested by the mood induction procedure. At the end of $5 \mathrm{~min}$, the investigator returned and stopped the music.

For the sad mood induction procedure, participants were instructed to imagine the death of someone they loved. Specific instructions were given to assist in this imagery, including picturing the loved one's last moments; details about his or her appearance, final words, and gestures; and unsuccessful attempts to comfort the loved one as he or she died. Instructions were given to focus on the death of this loved one and allow a sad mood to develop while the examiner was absent for $5 \mathrm{~min}$. During this time "Adagio for Strings, Op. 11" by Samuel Barber was played at half speed (Fox, Knight, \& Zelinski, 1998). Two additional scripts with guided imagery further elaborating on the loss of the loved one were administered at the one-third and two-thirds points in the testing session to help participants maintain a sad mood.

The neutral mood induction procedure was designed to match the negative mood induction procedure as closely as possible 


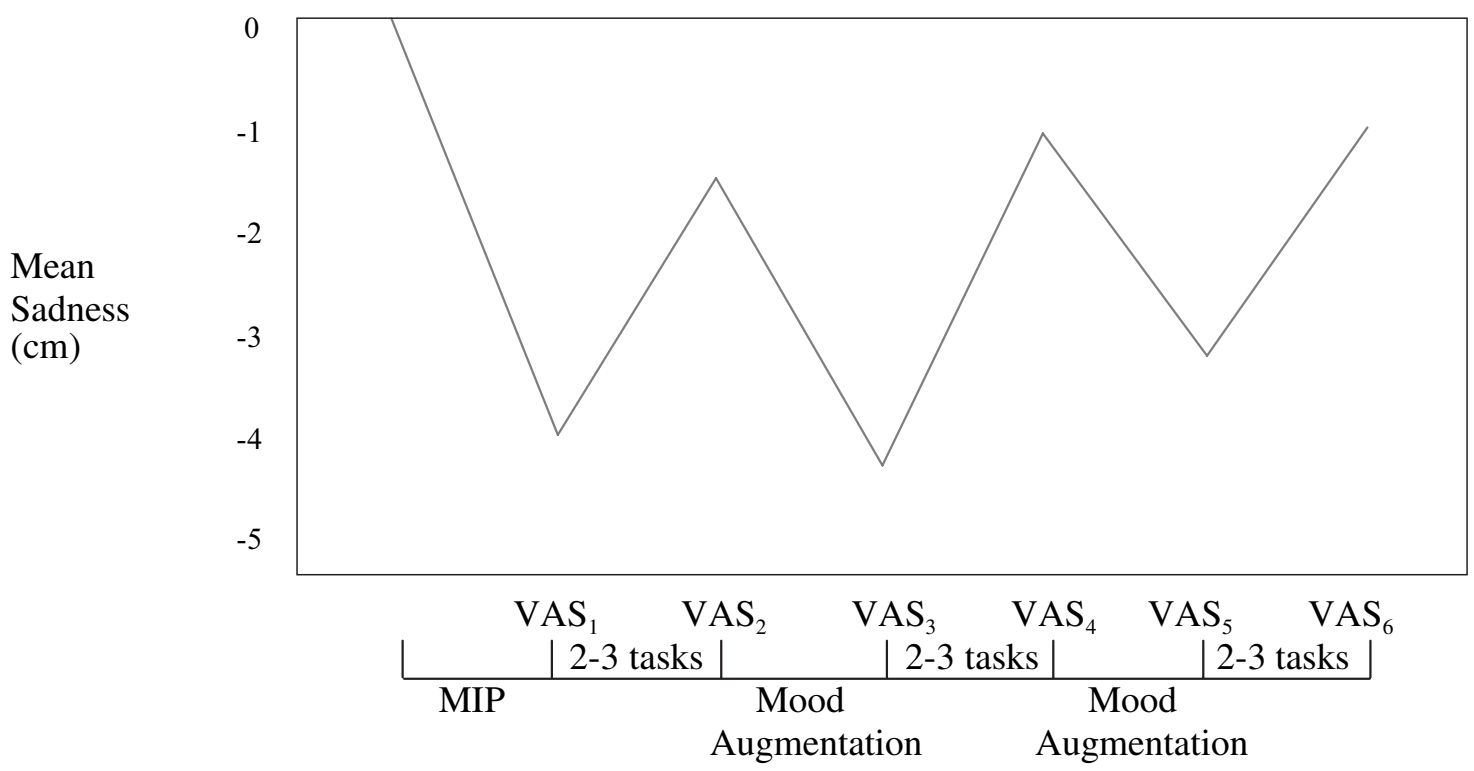

Figure 1. Average sadness ratings throughout the course of the sad condition session, measured in centimeter deviations from incoming mood on a visual analog scale (VAS). MIP $=$ mood induction procedure.

except for the valence of the induced mood. Accordingly, participants were instructed to vividly think about making a trip to the grocery store. The examiner followed a script for each participant that included specific instructions to imagine the details of the store and specific acts involved in grocery shopping. The examiner left the room after giving instructions to continue thinking about this experience. Kraftwerk's "Pocket Calculator" played during the course of the mood induction, which lasted 5 min. As with the sad mood induction, two neutral mood boosters, consisting of instructions to again conjure up the image of shopping for groceries, were also administered.

Mood assessment. Participants' mood ratings were recorded on visual analog scales (VAS) consisting of a 10 -cm line with better and worst printed at opposite ends, with a small line marked today bisecting the middle of the line. They were instructed that the bisecting line represented their mood before beginning the experiment and that it should serve as a reference point for all successive mood ratings for that session. All VAS mood ratings were measured in centimeters from the today mark.

Participants were tested individually in two sessions separated by 1 week. During the initial evaluation, informed consent was obtained from participants and the general procedure was explained. Medical and psychiatric histories were obtained, as well as demographic information regarding birthdate, years of education, primary language, handedness, and gender. Participants then performed practice trials for the cognitive tasks. The sad or neutral mood induction procedure was then administered, $\mathrm{VAS}_{1}$ was recorded, and two to three of the cognitive tests (depending on the counterbalancing order of the tests and their individual durations) were administered, lasting approximately $10-15 \mathrm{~min}$. $\mathrm{VAS}_{2}$ was then recorded and the first mood booster given, followed by $\mathrm{VAS}_{3}$. Participants performed another two to three tasks (10-15 min), recorded $\mathrm{VAS}_{4}$, received another mood booster, followed by $\mathrm{VAS}_{5}$, finished the last two or three tasks, and recorded $\mathrm{VAS}_{6}$ (see
Figure 1). At this point, the experimenter offered participants a brief optional writing exercise designed to further improve mood. Participants were debriefed after the second test session.

To minimize time lost to instruction and practice once a mood had been induced, participants practiced all tasks before the mood induction procedure. Music was turned off while participants performed the tasks. The seven tasks were given in one of seven orders, created by successively moving the task in first place to last place and making the second task first, the third second, and so on, as a means to attenuate possible effects of mood dissipation and fatigue. Individual participants performed the tasks in the same order for Sessions 1 and 2.

\section{Neurocognitive Tasks}

All tasks were run in Psyscope 1.2.5 on a Macintosh OS 9.2 system except for the Digit Span measure, which was conducted according to the WAIS procedure (Wechsler, 1997), with performance recorded manually by the experimenter. Words used in the emotion-processing tasks were chosen from M. Bradley and Lang (1999). Emotional and neutral word pairs were matched as closely as possible for valence, arousal, concreteness, frequency, and word length. Because the number of sad words that could be equated on these measures was limited and because of the large number of sad words required for the task battery, some threat- and traumarelated words were also used as "negative" stimuli. The percentage of depression- and trauma-related words are included in the individual task descriptions. Pilot testing ensured that task difficulty was sufficient to prevent ceiling or floor effects, and two different sets of stimuli were used for Test Sessions 1 and 2 .

The task battery included measures of prefrontal/executive function and limbic contributions to perception, attention and memory. Two measures of prefrontal/executive function, working memory 
and cognitive control, were tested using paired tasks of verbal and nonverbal stimuli.

\section{Working Memory Tasks}

Object 2-back. A standard 2-back paradigm, adapted from Postle, Jonides, Smith, Corkin, and Growdon (1997), was used with random polygons selected for low verbalizability. Images were displayed for $1 \mathrm{~s}$ with a 5-s interstimulus interval, with a total of 76 trials and 11 2-back matches. Participants were asked to respond "yes" or "no" with a key press to indicate whether or not each shape matched that which appeared two shapes ago. Performance was measured as percentage of correct responses.

Digit Span. A standard Digit Span measure was taken from the WAIS (Wechsler, 1997). Participants repeated number strings as read by the examiner and then in their reverse order. The number of digits to be recalled increased incrementally every two trials, and the task was terminated when the participant failed to correctly recall two trials consisting of the same number of digits. Total number correct was used as the dependent measure.

\section{Cognitive Control Tasks}

Stroop Color-Word Interference. Words were presented one at a time in the center port of a computer screen in a standard color Stroop experiment. Names of colors (e.g., red) or sizes (e.g., small) appeared in 24-point Chicago font in one of five colors on a black screen, and participants were instructed to name aloud the color in which each word appeared. One hundred words were presented in each of two blocks of trials. Reaction times (RTs) were recorded and the measure of interest, the Stroop interference effect, was calculated by subtracting each participant's mean RT on neutral trials from that on incongruent trials.

Go/No-Go. Numbers were displayed one at a time every 1,000 $\mathrm{ms}$ in the center of a computer screen. Participants were instructed to hit the space bar as quickly as possible in response to any number except "4." Two hundred ten numbers were presented, 30 of which were "4" and hence opportunities for false alarms. Numbers appeared in 100-point Chicago font in black on a white screen. Performance was measured as false alarm rate.

\section{Attention, Perception, and Memory for Affectively Valenced Materials}

Attention probe. Each trial began with a fixation signal, replaced after $1,000 \mathrm{~ms}$ by a pair of words consisting of a neutralnegative or a neutral-positive word pair. Emotional-neutral word pairs were displayed $4 \mathrm{~cm}$ apart vertically in the center of a computer screen for $500 \mathrm{~ms}$. After $500 \mathrm{~ms}$, both words disappeared and an "X" or an "N" appeared in the same location as that previously occupied by either the neutral or the emotional word. Participants were instructed to press the " $\mathrm{X}$ " or " $\mathrm{N}$ " keys in response to this probe. Congruent conditions consisted of the emotional word and the probe appearing in the same location; incongruent conditions consisted of the neutral word and the probe appearing in the same location. Negative words consisted of $50 \%$ depression-related words (e.g., gloom) and 50\% threat- or traumarelated words (e.g., brutal). There were 100 trials total, with equal numbers of congruent and incongruent, positive and negative pairs. Words were displayed in 24-point Chicago font in black on a white background. Response time was recorded and used to calculate a measure of attention bias for each participant. This measure was a difference between two differences, the first being the difference between the median RTs for congruent and incongruent trials with negative words, and the second being the same difference for trials with positive words. The more attention is directed to the locations of negative words, relative to positive words, the higher the value of this bias measure.

Free recall and recognition memory. Three sets of 16 words each were displayed, 1 word at a time for $2 \mathrm{~s}$ in the center of the screen. Half of the words had negative emotional valence and half had neutral valence. The negative words were $80 \%$ depressionrelated words and $20 \%$ threat- or trauma-related words. After each set was completed, participants were asked to recall as many of those 16 words as possible. After viewing all three sets, participants were shown a final list including these sets plus an equal number of new words in the recognition memory portion of the task. Participants were shown the words one at a time and asked whether the word was in one of the original sets. Words appeared in 36-point Helvetica font, in black on a white background. Recall and recognition performance were both measured as percentage correct.

Facial emotion recognition. This task was constructed using the Ekman photographs of people displaying neutral facial expressions, as well as the emotions happiness, sadness, anger, and fear (Matsumoto \& Ekman, 1988). Eighty faces were presented one at a time in the center of the computer screen for a fixed duration of $500 \mathrm{~ms}$. Each face was followed by a fixation cross that appeared until the participant's voice response triggered the next face to appear. Participants were instructed to choose one of five possible emotions (angry, afraid, neutral, sad, or happy) to describe the expression on each face. Accuracy of spoken response was the dependent measure.

\section{Results}

Participants whose performance on a particular task was greater than 2.5 standard deviations from the mean were removed from analysis for that task, resulting in an average of 1 participant removed per task $(M=1, S D=1$, range $=0-2)$. Mean RTs for each participant in each task were calculated excluding RTs greater than 2.5 standard deviations from the participant's mean. Relatively few data points were removed $(M=3.65, S D=1.72$, range $=0-8$ data points per participant per task), and these most often reflected errors in voice detection by the Psyscope microphone ( $\mathrm{RT}<80 \mathrm{~ms}$ or RT $>5,000 \mathrm{~ms}$ ) on the Stroop and facial emotion identification tasks.

In overview, we had three main goals in the analyses to be reported. First, we tested the effect of the mood induction procedures on mood in the two testing sessions as measured by the VAS. Second, we sought to determine, for each cognitive system, whether participants' moods influenced their performance. Statistically, this was addressed using matched pairs $t$ tests comparing performance in the neutral and negative mood conditions. Given our a priori hypotheses concerning the direction of the effects, we report one-tailed $t$ statistics. Third, we sought to determine whether the effect of mood was significantly more pronounced on some cognitive processes than on others. For this, we conducted a 
repeated measures analysis of variance (ANOVA) on standardized performance measures in each task, considering both mood and task as orthogonal factors, to test for a significant interaction between the two factors.

\section{Mood Ratings}

Mood measures were determined using the distance (in centimeters) between $\mathrm{VAS}_{0}$ and $\mathrm{VAS}_{1-6}$. A significant difference was found between the mean mood (averaged over $\mathrm{VAS}_{1-6}$ ) for the neutral mood induction procedure $(M=0.42, S D=0.99$, range $=$ -3.5 to 7$)$ and sad mood induction procedure $(M=-2.37, S D=$ 1.68 , range $=-11.3$ to 4$), t(32)=8.72, p<.001$, and at comparisons for each time point ( $p<.001$ in all cases). During the sad mood session, most participants experienced an improvement in mood between mood boosters but did not return to baseline during the testing session. This pattern contrasted with that observed for neutral mood ratings $\left(\mathrm{VAS}_{1-6}\right)$, which did not statistically differ from baseline. This analysis confirmed the Neutral and Sad mood induction procedures successfully induced the desired mood and the mood was sustained throughout the course of cognitive testing. Figure 1 shows the average mood ratings at each measurement point in the Sad mood conditions.

\section{Task Performance}

The analyses that follow were aimed at answering two questions: First, how did sad mood, compared with neutral mood, affect the cognitive processes required by the task battery? Second, given the striking disparity between the number of published reports of mood effects on memory bias and facial emotion perception on one hand and on other neurocognitive systems on the other hand, did the effects of mood vary across cognitive processes?

Sad mood caused a highly significant bias in recognition memory toward negatively valenced words relative to the neutral mood condition, $t=4.77, p<.0001$, as calculated by percentage correct for negative words divided by overall percentage correct. This bias ratio was $87 \%$ and $92 \%$ in the neutral and sad conditions, respectively. The recognition memory effect was purely one of bias; overall word recognition was not affected by mood, $t=-0.950$, $p=.175$, with $84 \%$ and $85 \%$ overall recognition for neutral and sad mood conditions. The mood bias effect found for recognition did not extend to recall, $t=-0.104, p=.459$, with $50 \%$ of negative words recalled in both conditions.

Sad mood also interfered with facial emotion recognition. Participants were more accurate in the neutral than in the sad mood condition, $t=2.62, p=.0069$, with mean accuracies of $84 \%$ and $81 \%$, respectively. In addition to examining whether participants would exhibit a general impairment in facial emotion recognition in a sad mood relative to a neutral mood, we also explored the possibility that sad mood would affect this function in a more specific way, namely through a systematic bias to mislabel happy faces as neutral or sad, or neutral faces as sad. To that end, for each participant in each condition, we tallied the number of errors consistent with this type of bias. As in the majority of studies with depressed patients, there was no such bias, $t=0.5, p=.31$, with mean rates of negatively biased errors of $18 \%$ and $14 \%$ in the neutral and sad conditions, respectively.
Sad mood did not bias attention toward negative words. Indeed, we found a nonsignificant trend in the opposite direction for our measure of attention bias, $t=-1.23, p>0.230$, two-tailed, with a mean RT measure of $-10.3 \mathrm{~ms}$ and $-14.5 \mathrm{~ms}$ in the neutral and sad mood conditions, respectively. We also examined the effect of sad mood on negative bias within the neutral-negative word pairs alone and also failed to find an effect, $t=.14, p>.445$, with means of $2.2 \mathrm{~ms}$ and $-3.6 \mathrm{~ms}$ for the neutral and sad mood conditions, respectively.

Sad mood did not affect performance on any of the executive function tasks. For the Digit Span task, participants' total number correct was 20.5 and 20.7 in the neutral and sad conditions, respectively, and did not differ, $t=0.335, p>.1$. The other working memory task, Object 2-Back, also failed to show any effects of mood, $t=0.135, p>.1$, with a mean accuracy of $87 \%$ in both conditions. The two cognitive control tasks were similarly unaffected by mood. The mean Stroop effect size in the neutral and sad mood conditions was $114 \mathrm{~ms}$ and $115 \mathrm{~ms}$, respectively, which did not differ significantly between conditions, $t=0.445, p=.660$, for the Stroop task. Accuracy was also not affected by mood for the Go/No-Go task, $t=$ $-0.423, p>.676$, with mean false alarm rates of $19.6 \%$ and $18.8 \%$ in the neutral and sad conditions, respectively.

The final analysis reported here concerns the pattern of mood effects over tasks. In contrast to studies of depressed patients, studies of normal participants have so far revealed quite limited effects of sad mood on cognitive abilities, largely confined to memory bias. However, we cannot know whether the broader set of abilities has simply not yet been studied or whether they have been studied and produced null results. Having administered a diverse set of tasks, tapping the range of neurocognitive functions affected by depression in the same group of normal participants in sad and neutral moods, we can now address this question. Specifically, we can ask whether mood affected cognition unevenly in this study. That is, more than simply showing that some abilities were not measurably affected by sad mood and others were, we can find out whether some were reliably less affected by mood than others.

To address this question, we transformed the measures of performance in the different tasks to a common scale by calculating $z$ scores for each participant's performance in each mood condition in each task, defined relative to the distribution of neutral condition scores for each task. The $z$ scores were then submitted to a two-way repeated measures ANOVA with factors mood (sad or neutral) and task (the seven different tasks). This analysis revealed a significant interaction between mood and task, $F(6,24)=3.394$, $p=.014$. In other words, sad mood did not merely influence performance in some tasks significantly and in others not; instead, mood was significantly more influential on some tasks than others. This corresponds visually to a significantly jagged, rather than flat, profile of mood effects over tasks. Figure 2 illustrates this by showing the effect of sad mood in terms of the disparity between mean performance in the neutral and negative mood conditions, measured in terms of standard deviations of performance in the neutral condition.

\section{Discussion}

Cognitive psychologists are generally careful to control, or at least measure, the age, gender, and native language of participants (for studies with verbal materials) and take exquisite care to 


\section{Mood Effects by Task}

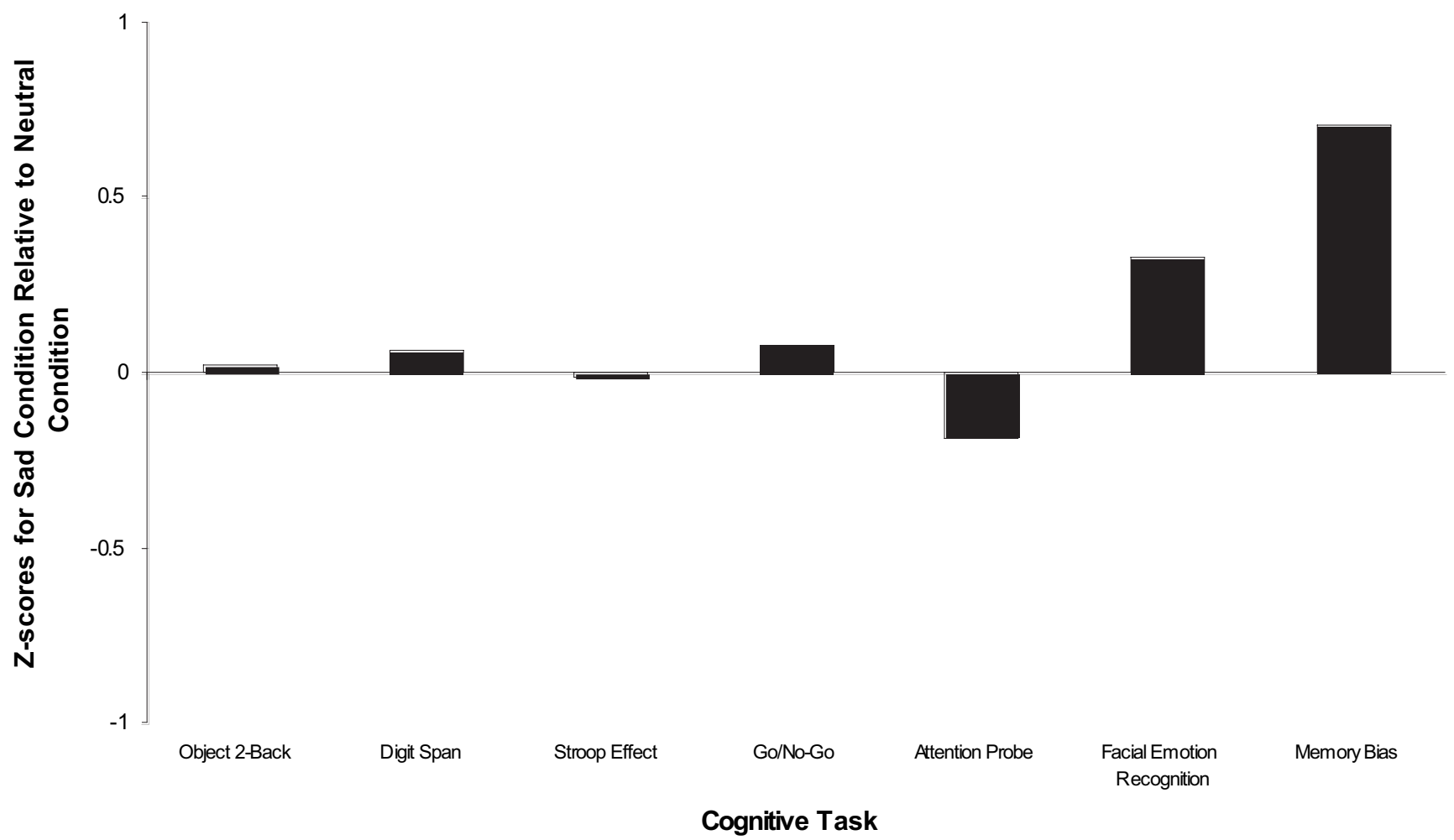

Figure 2. Profile of sad mood effect across tasks, with positive difference scores indicating that sadness caused impaired performance (executive tasks) or more negatively biased performance (attention, perception and memory tasks).

control the attentional states of participants during experiments through task instructions, quiet testing rooms, and, sometimes, performance incentives. In contrast, mood state is rarely measured in a typical cognitive psychology experiment. Most of what we know about cognition therefore comes from experiments in which the majority of participants can be presumed to be in a "neutral" or average mood, but the specific nature of participants' moods is neither controlled nor measured. As a result, relatively little is known about the effects of mood on cognition, despite the fact that mood is a salient and pervasive feature of our mental lives. The present study was motivated by the dearth of evidence on the relation between mood and cognition in normal healthy people and sought to address a basic question about the influence of a particular type of mood, sadness, on cognition.

As noted earlier, there are good reasons to expect a pattern of extensive influence of sad mood on cognition. Anatomically, many of the brain regions subserving perception, attention, memory, and executive function are influenced by mood, becoming either more or less active in sad mood compared with neutral mood. In addition, a large literature on cognition in depression documents executive dysfunction and changes in attention, perception, and memory in patients suffering from depression. However, the literature on mood and cognition in healthy participants does not show a similar pattern of a pervasive effect of sad mood. Instead, one finds ample evidence of sad mood effects on memory, specifically a mood-congruent memory bias, but little or no evidence of other effects. The literature contains relatively few studies of sad mood on other cognitive processes, and isolated positive findings are balanced by a greater number of published null results.

There are two possible interpretations for this unexpected pattern of results in the literature. One is that studies of sad mood have so far been undertaken primarily with memory for affectively valenced material and that the dearth of reported sad mood effects on facial emotion recognition, attention, working memory, and inhibitory control simply reflects the fact that the relevant experiments have rarely or never been carried out. The other possibility is that such experiments have been carried out but produced null results, most of which went unpublished. In any individual case of a null result, one would not know whether the absence of an effect is due to the encapsulation of the cognitive process in question from sad mood or to inadequacies of the experiment, presenting an obstacle to publication.

Distinguishing between these possibilities requires an experimental design in which the same mood induction procedure, in the same sample of participants, is used to assess mood effects across an array of cognitive processes. This is what was done in the present study. The results of this study indicate a very circumscribed effect of sad mood on cognition, influencing affective memory bias and facial emotion recognition only. The same mood induction procedure, administered to the same participants in the 
same sessions, failed to affect performance on a spatial attention task and four executive function tasks. This is consistent with published results and with the hypothesis that earlier studies of sad mood effects on attention and executive function have probably gone unreported because of the ambiguity of null results.

Of course, it is always possible that a better mood induction procedure, or more or better tests of attention and executive function, would have revealed effects of sadness on attention and executive function. Perhaps the particular tasks we used were insufficiently sensitive measures of executive function and attention. We cannot rule this possibility out, yet it is unlikely for two reasons. First, the relevant performance measures for some of the tasks had no ceiling (Digit Span, Stroop Color-Word, attention probe), and for those that did, participants were not at ceiling or floor on any of them, by the criterion of having at least 1 standard deviation between the mean and the highest or lowest possible scores. Second, as noted in the introduction, positive findings have been reported in the depression literature for the types of tasks we used, specifically Stroop Color-Word, Go/No-Go, Object 2-Back, Digit Span, and attention probe. Alternatively, perhaps a more extreme sad mood than that achieved in our study would influence a greater range of cognitive processes. Even so, on the basis of the present results we can conclude that a degree of sadness sufficient to influence affective memory bias and facial emotion recognition has no measurable effect on the other cognitive processes tested and that this was true of executive function tested with four different tasks.

How do the present results inform our understanding of cognition in depression? They suggest that the cognitive impairments and biases in depression may not simply be the result of the disease's hallmark, sad mood. One possibility is that the cognitive aspects of depression are an independent component of the disease. Although laypeople generally think of depression as defined solely by sad mood, it is in fact a complex disease affecting energy level, psychomotor speed, concentration, sleep, appetite, and libido, as well as mood (Diagnostic and Statistical Manual of Mental Disorders, fourth ed.; American Psychiatric Association, 1994). The finding that cognitive improvements may precede mood recovery during therapy for depression (Dunkin et al., 2000) lends support to this interpretation.

It is also possible that there are individual differences in the effects of sad mood on cognition. It has been suggested that one determinant of vulnerability to depression is the longevity of the effects of sad mood on cognition (Gilboa et al., 1997). A related idea is that vulnerability is associated with a more pervasive effect of sad mood on cognition (Miranda \& Persons, 1988). Perhaps in vulnerable individuals a sad mood is capable of biasing attention and perception as well as memory and interfering with executive functions. This set of changes could, in themselves, contribute to the onset of a depressive episode. It is likely that few participants in the present study were vulnerable to depression because a history of depression was an exclusionary criterion.

The conclusion of this study, that normal sadness has an extremely circumscribed effect on cognition, does not necessarily generalize to mood in general. There is reason to believe that sadness and happiness are not simply opposite ends of a single mood continuum but instead represent independent dimensions of mood (Dalgleish, 2004). Indeed, positive mood has been demonstrated to influence executive function (Bartolic, Basso, Schefft,
Glauser, \& Titanic-Schefft, 1999; L. H. Phillips, Bull, Adams, \& Fraser, 2002) and has been shown to do so even when negative mood does not (Oaksford, Grainger, Morris, \& Williams, 1996), presumably via its effects on the neurotransmitter dopamine (Ashby, Isen, \& Turken, 1999). However, for sadness the results of this study suggest fairly limited effects of mood. The cognitive processing of affectively valenced words and faces was found to be permeable to the mood state of sadness, but cognition more generally appeared relatively encapsulated from sadness, at least in young adults with no history of depression.

\section{References}

Aalto, S., Naatanen, P., Wallius, E., Metsahonkala, L., Stenman, H., Niem, P. M., et al. (2002). Neuroanatomical substrata of amusement and sadness: A PET activation study using film stimuli. NeuroReport, 13(1), $67-73$.

Algom, D., Chajut, E., \& Lev, S. (2004). A rational look at the emotional Stroop phenomenon: A generic slowdown, not a Stroop effect. Journal of Experimental Psychology: General, 133, 323-338.

American Psychiatric Association. (1994). Diagnostic and statistical manual of mental disorders (4th ed.). Washington, DC: Author.

Ashby, F. G., Isen, A. M., \& Turken, A. U. (1999). A neuropsychological theory of positive affect and its influence on cognition. Psychological Review, 106, 529-550.

Austin, M. P., Mitchell, P., Wilhelm, K., Parker, G., Hickie, I., Brodaty, H., et al. (1999). Cognitive function in depression: A distinct pattern of frontal impairment in melancholia? Psychological Medicine, 29, 73-85.

Bartolic, E. I., Basso, M. R., Schefft, B. K., Glauser, T., \& Titanic-Schefft, M. (1999). Effects of experimentally-induced emotional states on frontal lobe cognitive task performance. Neuropsychologia, 37, 677-683.

Bench, C. J., Friston, K. J., Brown, R. G., Scott, L. C., Frackowiak, R. S., \& Dolan, R. J. (1992). The anatomy of melancholia-Focal abnormalities of cerebral blood flow in major depression. Psychological Medicine, 22, 607-615.

Bouhuys, A. L., Bloem, G. M., \& Groothuis, T. G. (1995). Induction of depressed and elated mood by music influences the perception of facial emotional expressions in healthy subjects. Journal of Affective Disorders, 33(4), 215-226.

Bouhuys, A. L., Geerts, E., \& Gordijn, M. C. (1999). Depressed patients' perceptions of facial emotions in depressed and remitted states are associated with relapse: A longitudinal study. Journal of Nervous and Mental Disease, 187, 595-602.

Bouhuys, A. L., Geerts, E., \& Mersch, P. P. (1997). Relationship between perception of facial emotions and anxiety in clinical depression: Does anxiety-related perception predict persistence of depression? Journal of Affective Disorders, 43, 213-223.

Bower, G. H., \& Forgas, J. P. (2001). Handbook of affect and social cognition. Mahwah, NJ: Erlbaum.

Bradley, B. P., Mogg, K., \& Lee, S. C. (1997). Attentional biases for negative information in induced and naturally occurring dysphoria. Behaviour Research and Therapy, 35, 911-927.

Bradley, M. M., \& Lang, P. J. (1999). Affective norms for English words $(A N E W)$. Gainesville, FL: NIMH Center for the Study of Emotion and Attention, University of Florida.

Braver, T. S., Cohen, J. D., Nystrom, L. E., Jonides, J., Smith, E. E., \& Noll, D. C. (1997). A parametric study of prefrontal cortex involvement in human working memory. NeuroImage, 5, 49-62.

Bush, G., Luu, P., \& Posner, M. I. (2000). Cognitive and emotional influences in anterior cingulate cortex. Trends in Cognitive Sciences, 4 , 215-222.

Cabeza, R., \& Nyberg, L. (2000). Imaging cognition II: An empirical review of 275 PET and fMRI studies. Journal of Cognitive Neuroscience, 12, 1-47. 
Casey, B. J., Cohen, J. D., O’Craven, K., Davidson, R. J., Irwin, W., Nelson, C. A., et al. (1998). Reproducibility of fMRI results across four institutions using a spatial working memory task. NeuroImage, 8, 249261.

Cohen, J. D., Perlstein, W. M., Braver, T. S., Nystrom, L. E., Noll, D. C., Jonides, J., et al. (1997, April 10). Temporal dynamics of brain activation during a working memory task. Nature, 386, 604-608.

Cooley, E. L., \& Nowicki, S., Jr. (1989). Discrimination of facial expressions of emotion by depressed subjects. Genetic, Social, and General Psychology Monographs, 115(4), 449-465.

Dalgleish, T. (2004). The emotional brain. Nature Reviews Neuroscience, $5,582-589$

Davidson, R. J., Pizzagalli, D., Nitschke, J. B., \& Putnam, K. (2002). Depression: Perspectives from affective neuroscience. Annual Review of Psychology, 53, 545-574.

Drevets, W. C. (1999). Prefrontal cortical-amygdalar metabolism in major depression. Annals of the New York Academy of Sciences, 877, 614637

Drevets, W. C. (2003). Neuroimaging abnormalities in the amygdala in mood disorders. Annals of the New York Academy of Sciences, 985, $420-444$

Drevets, W. C., Videen, T. O., Price, J. C., Preskorn, S. H., Carmichael, S. T., \& Raichle, M. E. (1992). A functional anatomical study of unipolar depression. Journal of Neuroscience, 12, 3628-3641.

Dunkin, J. J., Leuchter, A. F., Cook, I. A., Kasl-Godley, J. E., Abrams, M., \& Rosenberg-Thompson, S. (2000). Executive dysfunction predicts nonresponse to fluoxetine in major depression. Journal of Affective Disorders, 60, 13-23.

Eugene, F., Levesque, J., Mensour, B., Leroux, J. M., Beaudoin, G., Bourgouin, P., et al. (2003). The impact of individual differences on the neural circuitry underlying sadness. NeuroImage, 19, 354-364.

Feinberg, T. E., Rifkin, A., Schaffer, C., \& Walker, E. (1986). Facial discrimination and emotional recognition in schizophrenia and affective disorders. Archives of General Psychiatry, 43, 276-279.

Fiedler, K., Nickel, S., Muehlfriedel, T., \& Unkelbach, C. (2001). Is mood congruency and effect of genuine memory or response bias? Journal of Experimental Social Psychology, 37, 201-214.

Fox, L. S., Knight, B. G., \& Zelinski, E. M. (1998). Mood induction with older adults: A tool for investigating effects of depressed mood. Psychology and Aging, 13, 519-523.

Gemar, M. C., Kapur, S., Segal, Z. V., Brown, G. M., \& Houle, S. (1996). Effects of self-generated sad mood on regional cerebral activity: A PET study in normal subjects. Depression, 4, 81-88.

Gilboa, E., Roberts, J. E., \& Gotlib, I. H. (1997). The effects of induced and naturally occurring dysphoric mood on biases in self-evaluation and memory. Cognition \& Emotion, 11, 65-82.

Gilboa-Schechtman, E., Revelle, W., \& Gotlib, I. H. (2000). Stroop interference following mood induction: Emotionality, mood congruence and concern relevance. Cognitive Therapy and Research, 24, 491-502.

Gur, R. C., Erwin, R. J., Gur, R. E., Zwil, A. S., Heimberg, C., \& Kraemer, H. C. (1992). Facial emotion discrimination: II. Behavioral findings in depression. Psychiatry Research, 42, 241-251.

Harvey, P. O., Le Bastard, G., Pochon, J. B., Levy, R., Allilaire, J. F., Dubois, B., et al. (2004). Executive functions and updating of the contents of working memory in unipolar depression. Journal of Psychiatric Research, 38, 567-576.

Haxby, J. V., Hoffman, E. A., \& Gobbini, M. I. (2002). Human neural systems for face recognition and social communication. Biological Psychiatry, 51, 59-67.

Kennedy, S. J., Evans, K. R., Kruger, S., Mayberg, H., Meyer, J. H., McCann, S., et al. (2001). Changes in regional brain glucose metabolism measured with positron emission tomography after paroxetine treatment of major depression. American Journal of Psychiatry, 158, 899-905.

Kiehl, K. A., Liddle, P. F., \& Hopfinger, J. B. (2000). Error processing and the rostral anterior cingulate: An event-related fMRI study. Psychophysiology, 37, 216-223.

Lane, R. D., Reiman, E. M., Ahern, G. L., Schwartz, G. E., \& Davidson, R. J. (1997). Neuroanatomical correlates of happiness, sadness, and disgust. American Journal of Psychiatry, 154, 926-933.

Langenecker, S. A., Bieliauskas, L. A., Rapport, L. J., Zubieta, J. K., Wilde, E. A., \& Berent, S. (2005). Face emotion perception and executive functioning deficits in depression. Journal of Clinical and Experimental Neuropsychology, 27, 320-333.

Leppanen, J. M., Milders, M., Bell, J. S., Terriere, E., \& Hietanen, J. K. (2004). Depression biases the recognition of emotionally neutral faces. Psychiatry Research, 128, 123-133.

Levesque, J., Eugene, F., Joanette, Y., Paquette, V., Mensour, B., Beaudoin, G., et al. (2003). Neural circuitry underlying voluntary suppression of sadness. Biological Psychiatry, 53, 502-510.

Liddle, P. F., Kiehl, K. A., \& Smith, A. M. (2001). Event-related fMRI study of response inhibition. Human Brain Mapping, 12, 100-109.

Lim, S. L., \& Kim, J. H. (2005). Cognitive processing of emotional information in depression, panic, and somatoform disorder. Journal of Abnormal Psychology, 114, 50-61.

Liotti, M., Mayberg, H. S., Brannan, S. K., McGinnis, S., Jerabek, P., \& Fox, P. T. (2000). Differential limbic-cortical correlates of sadness and anxiety in healthy subjects: Implications for affective disorders. Biological Psychiatry, 48, 30-42.

MacLeod, C., Mathews, A., \& Tata, P. (1986). Attentional bias in emotional disorders. Journal of Abnormal Psychology, 95, 15-20.

Marvel, C. L., \& Paradiso, S. (2004). Cognitive and neurological impairment in mood disorders. Psychiatric Clinics of North America, 27, $19-36$.

Mathews, A., Ridgeway, V., \& Williamson, D. A. (1996). Evidence for attention to threatening stimuli in depression. Behaviour Research and Therapy, 34, 695-705.

Matsumoto, \& Ekman, P. (1988). Japanese and Caucasian facial expressions of emotion (JACFEE) [slides]. San Francisco, CA: Intercultural and Emotion Research Laboratory, Department of Psychology, San Francisco State University.

Mayberg, H. S., Liotti, M., Brannan, S. K., McGinnis, S., Mahurin, R. K., Jerabek, P. A., et al. (1999). Reciprocal limbic-cortical function and negative mood: Converging PET findings in depression and normal sadness. American Journal of Psychiatry, 156, 675-682.

McCabe, S. B., Gotlib, I. H., \& Martin, R. A. (2000). Cognitive vulnerability for depression: Deployment of attention as a function of history of depression and current mood state. Cognitive Therapy and Research, 24, 427-444.

McKenna, F. P., \& Sharma, D. (2004). Reversing the emotional Stroop effect reveals that it is not what it seems: The role of fast and slow components. Journal of Experimental Psychology: Learning, Memory, and Cognition, 30, 382-392.

Mikhailova, E. S., Vladimirova, T. V., Iznak, A. F., Tsusulkovskaya, E. J., \& Sushko, N. V. (1996). Abnormal recognition of facial expression of emotions in depressed patients with major depression disorder and schizotypal personality disorder. Biological Psychiatry, 40, 697-705.

Miranda, J., \& Persons, J. B. (1988). Dysfunctional attitudes are moodstate dependent. Journal of Abnormal Psychology, 97, 76-79.

Mogg, K., Mathews, A., Eysenck, M., \& May, J. (1991). Biased cognitive operations in anxiety: Artifact, processing priorities or attentional search? Behaviour Research and Therapy, 29, 459-467.

Moritz, S., Birkner, C., Kloss, M., Jahn, H., Hand, I., Haasen, C., et al. (2002). Executive functioning in obsessive-compulsive disorder, unipolar depression, and schizophrenia. Archives of Clinical Neuropsychology, 17, 477-483.

Murphy, F. C., Nimmo-Smith, I., \& Lawrence, A. D. (2003). Functional neuroanatomy of emotions: A meta-analysis. Cognitive, Affective, and Behavioral Neuroscience, 3, 207-233. 
Oaksford, M., Grainger, B., Morris, F., \& Williams, J. M. (1996). Mood, reasoning and central executive processes. Journal of Experimental Psychology: Learning, Memory and Cognition, 22, 477-493.

Perez, M. G., Rivera, R., Fuster, A. B., \& Rodriguez, A. R. (1999). Attentional biases and vulnerability to depression. Spanish Journal of Psychology, 2(1), 11-19.

Peterson, B. S., Skudlarski, P., Gatenby, J. C., Zhang, H., Anderson, A. W., \& Gore, J. C. (1999). An fMRI study of Stroop word-color interference: Evidence for cingulate subregions subserving multiple distributed attentional systems. Biological Psychiatry, 45, 1237-1258.

Phan, K. L., Wager, T., Taylor, S. F., \& Liberzon, I. (2002). Functional neuroanatomy of emotion: A meta-analysis of emotion activation studies in PET and fMRI. NeuroImage, 16, 331-348.

Phelps, E. A., \& LeDoux, J. E. (2005, October 20). Contributions of the amygdala to emotion processing: From animal models to human behavior. Neuron, 48, 175-187.

Phillips, L. H., Bull, R., Adams, E., \& Fraser, L. (2002). Positive mood and executive function: Evidence from Stroop and fluency tasks. Emotion, 2, $12-22$.

Phillips, M. L., Drevets, W. C., Rauch, S. L., \& Lane, R. (2003a). Neurobiology of emotion perception I: The neural basis of normal emotion perception. Biological Psychiatry, 54, 504-514.

Phillips, M. L., Drevets, W. C., Rauch, S. L., \& Lane, R. (2003b). Neurobiology of emotion perception II: Implications for major psychiatric disorders. Biological Psychiatry, 54, 515-528.

Postle, B. R., Jonides, J., Smith, E. E., Corkin, S., \& Growdon, J. H.
(1997). Spatial, but not object, delayed response is impaired in early Parkinson's disease. Neuropsychology, 11, 171-179.

Rogers, R. D., Kasai, K., Koji, M., Fukuda, R., Iwanami, A., Nakagome, K., et al. (2004). Executive and prefrontal dysfunction in unipolar depression: A review of neuropsychological and imaging evidence. Neuroscience Research, 50, 1-11.

Seibert, P. S., \& Ellis, H. C. (1991). A convenient self-referencing mood induction procedure. Bulletin of the Psychonomic Society, 29, 121-124.

Smith, E. E., \& Jonides, J. (1997). Working memory: A view from neuroimaging. Cognitive Psychology, 33, 5-42.

Steele, J. D., \& Lawrie, S. M. (2004). Segregation of cognitive and emotional function in the prefrontal cortex: Stereotactic meta-analysis. NeuroImage, 21, 868-875.

Varner, L. J., \& Ellis, H. C. (1998). Cognitive activity and physiological arousal: Processes that mediate mood-congruent memory. Memory \& Cognition, 26, 939-950.

Wechsler, D. A. (1997). Wechsler Adult Intelligence Scale (3rd ed.). San Antonio, TX: Psychological Corporation.

Williams, J. M., Mathews, A., \& MacLeod, C. (1996). The emotional Stroop task and psychopathology. Psychological Bulletin, 120, 3-24.

Williams, J. M., Watts, F. M., Macleod, C., \& Mathews, A. (1997). Cognitive psychology and emotional disorders (2nd ed.). New York: Wiley.

Received November 13, 2006 Revision received April 2, 2007 Accepted June 18, 2007

\section{Correction to D'Argembeau and Van der Linden (2007)}

In the article "Facial Expressions of Emotion Influence Memory for Facial Identity in an Automatic Way” by Arnaud D’Argembeau and Martial Van der Linden (Emotion, 2007, Vol. 7, No. 3, pp. 507-515), the image printed for Figure 3 was incorrect. The correct image should be as follows:

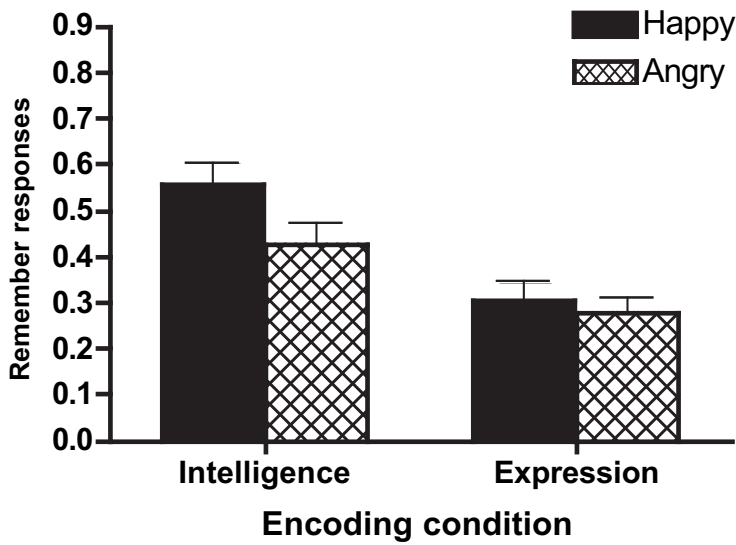

DOI: $10.1037 / 1528-3542.7 .4 .811$ 